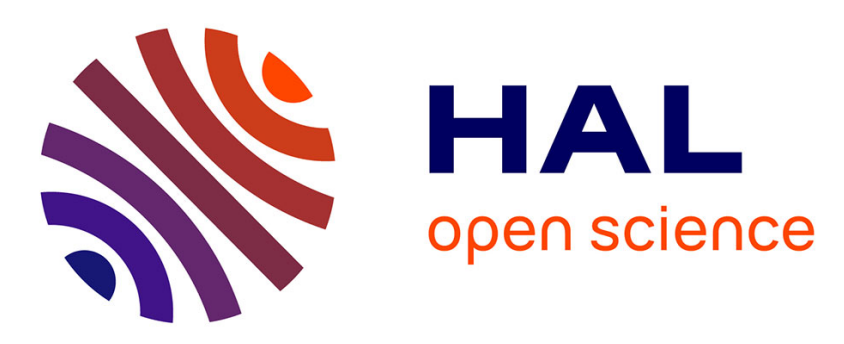

\title{
Subwavelength engineering for Brillouin gain optimization in silicon optomechanical waveguides
}

Jianhao Zhang, Omar Ortiz, Xavier Le Roux, Eric Cassan, Laurent Vivien, Delphine Marris-Morini, N. D. Lanzillotti-Kimura, Carlos Alonso-Ramos

\section{- To cite this version:}

Jianhao Zhang, Omar Ortiz, Xavier Le Roux, Eric Cassan, Laurent Vivien, et al.. Subwavelength engineering for Brillouin gain optimization in silicon optomechanical waveguides. Optics Letters, 2020, 45 (13), pp.3717. 10.1364/OL.397081 . hal-03028673

\section{HAL Id: hal-03028673 \\ https://hal.science/hal-03028673}

Submitted on 30 Nov 2020

HAL is a multi-disciplinary open access archive for the deposit and dissemination of scientific research documents, whether they are published or not. The documents may come from teaching and research institutions in France or abroad, or from public or private research centers.
L'archive ouverte pluridisciplinaire HAL, est destinée au dépôt et à la diffusion de documents scientifiques de niveau recherche, publiés ou non, émanant des établissements d'enseignement et de recherche français ou étrangers, des laboratoires publics ou privés. 


\title{
Subwavelength engineering for Brillouin gain optimization in silicon optomechanical waveguides
}

\author{
Jianhao Zhang, ${ }^{*}$ Omar Ortiz, Xavier le Roux, Eric Cassan, laurent Vivien, \\ DelPhine MarRis-Morini, Norberto Daniel Lanzillotti-Kimura, Carlos \\ ALONSO-RAMOS*
}

Université Paris-Saclay, CNRS, Centre de Nanosciences et de Nanotechnologies, 91120, Palaiseau, France. *Corresponding author: Jianhao.zhang@c2n.upsaclay.fr carlos.ramos@c2n.upsaclay.fr

Brillouin optomechanics has recently emerged as a promising tool to implement new functionalities in silicon photonics, including high-performance opto-RF processing and non-reciprocal light propagation. One key challenge in this field is to maximize the photon-phonon interaction and the phonon lifetime, simultaneously. Here, we propose a new strategy that exploits subwavelength engineering of the photonic and phononic modes in silicon membrane waveguides to maximize the Brillouin gain. By properly designing the dimensions of the subwavelength periodic structuration, we tightly confine near-infrared photons and $\mathrm{GHz}$ phonons, minimizing leakage losses and maximizing the Brillouin coupling. Our theoretical analysis predicts a high mechanical quality factor of up to 700 and a remarkable Brillouin gain yielding $3500(W \cdot m)^{-1}$ for minimum feature size of $50 \mathrm{~nm}$, compatible with electron-beam lithography. We believe that the proposed waveguide with subwavelength nanostructuration holds great potential for the engineering of Brillouin optomechanical interactions in silicon.

Brillouin scattering is a third-order optical nonlinear process that results from an optomechanical coupling [1]. This effect has been widely explored in the context of laser physics, microwavefrequency phonon excitation, and precision spectroscopy, among others [2]. Brillouin scattering process entails the excitation of mechanical waves, with frequencies ranging between a few tens of $\mathrm{MHz}$ and hundreds of $\mathrm{GHz}$, which are frequently referred to as acoustic phonons [3]. The availability of materials co-confining phonons and photons in the same device has enabled the demonstration of Brillouin scattering on chip, showing exceeding strengths over Kerr and Raman interactions [4-6]. There have been remarkable achievements in the control of on-chip Brillouin optomechanics using chalcogenides [4], gallium arsenide [7-9], and silicon $[10,11]$. Optical phonons with frequencies up to $10 \mathrm{GHz}[5]$ in nanometric-scale waveguides have wavelengths close to one micron, enable the strong interaction with photons in the near- infrared [11-15], remarkably large Brillouin gain [16] and Brillouin lasing [17] in silicon. While Brillouin scattering in optical fibers is mainly governed by the electrostriction effect in the bulk material, Brillouin scattering in nanophotonic silicon waveguides comprises a complex interplay between the bulk electrostriction and the geometry-dependent radiation pressure at the waveguide boundaries $[10,11,15,18]$. Different strategies have been proposed to optimize Brillouin gain in silicon waveguides $[13,17,19,20]$. Pedestal waveguides provide strong photon-phonon overlap [13] with a good Brillouin gain of up to $3000(\mathrm{~W} \cdot \mathrm{m})^{-1}$. However, minimizing phonon leakage towards the silica under-cladding requires a silica pedestal with a narrow width, which may be challenging to fabricate. Silicon membrane rib waveguides can achieve remarkably low photonic and phononic losses by implementing silicon air gaps that partially isolate the waveguide core and the lateral silicon slab $[16,17]$. Still, the photonic mode is mainly confined in the waveguide core while the phononic mode expands all over the slab. This results in a comparatively small photon-phonon overlap that limits the intra-mode Brillouin gain to $1000(\mathrm{~W} \cdot \mathrm{m})^{-1}[16]$. Theoretical calculations predicted a remarkably large Brillouin gain of $8000(\mathrm{~W} \cdot \mathrm{m})^{-1}$ in phoxonic crystals, exploiting bandgap confinement [20] of photons and phonons, simultaneously. Nevertheless, the performance of these phoxonic crystals may be hampered by the narrow bandwidth and high optical propagation loss, typically linked to bandgap confinement [21]. On the other hand, low optical loss and ultrawideband operation have been demonstrated in silicon subwavelength grating waveguides exploiting index-contrast confinement [22-25]. Silicon optomechanical waveguides using index-contrast confinement for photons and bandgap confinement for phonons have been recently proposed [19]. Such optomechanical waveguides could provide a Brillouin gain of $1750(\mathrm{~W} \cdot \mathrm{m})^{-1}$. Yet, the performance of the bandgap-confined phononic modes might be hampered by the intrinsically limited bandwidth of this approach and by loss arising from tight sensitivity to fabrication imperfections.

Here, we propose a new waveguide geometry that exploits subwavelength index-contrast engineering to confine optical 
modes and subwavelength mechanical softening to minimize the loss of the acoustic modes. By leveraging the nanostructuration, the proposed subwavelength-lattice waveguide allows to enhance simultaneously the photon-phonon interaction and increase the quality factor of the mechanical mode. Our calculations show that the proposed waveguide may provide Brillouin gain as high as $10000(\mathrm{~W} \cdot \mathrm{m})^{-1}$

As illustrated in Fig. 1 (a), the proposed membrane waveguide consists of a central strip and a lattice of lateral arms that anchor the waveguide core to the infinite silicon slab. The phonon confinement in our structure is achieved by a large impedance mismatch between the air and the silicon core, instead of a phononic bandgap, while the arm lattice with subwavelength period introduces a small mechanical dissipation. The core has a width of $w_{w g}=500 \mathrm{~nm}$ and a thickness of $t_{w g}=220 \mathrm{~nm}$, while the arms have a width of $w_{a}=$ $2.5 \mu \mathrm{m}$. The acoustic leakage is reduced by exploiting the height contrast at the core-nanoarm interface and nanorm-slab interface, where the spatial mismatch of acoustic mode causes a potential well (difference in eigen frequency) for a shared wavevector. To maximize this height contrast, we choose a nanoarm, thickness of $t_{a}=50 \mathrm{~nm}$. We refer "width" to dimension along $x$ while "length" to that along $z$. This width $(2.5 \mu \mathrm{m})$ is chosen to ensure a low optical loss, while avoiding acoustic resonance in nanoarms. An increase of this width causes slight variation in Brillouin gain and is not detailed here. We choose a period of $\Lambda=240 \mathrm{~nm}$, well below the acoustic wavelength (650 nm in silicon, at $9 \mathrm{GHz}$ ) and optical wavelength (640 $\mathrm{nm}$ in silicon, i.e., $1550 \mathrm{~nm}$ in vacuum).

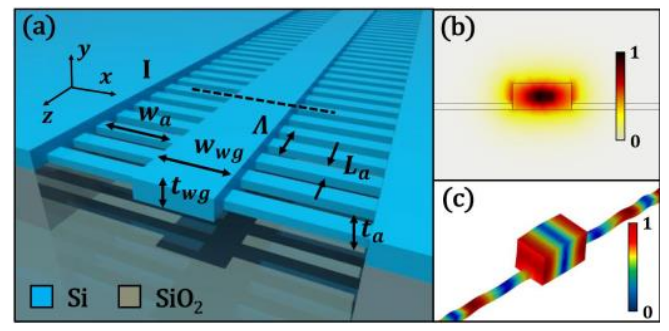

Fig. 1. (a) Schematic of the proposed optomechanical silicon waveguide based on a subwavelength lattice. Optimized dimensions: $t_{a}=50 \mathrm{~nm}$, $t_{w g}=220 \mathrm{~nm}, w_{w g}=500 \mathrm{~nm}$ and $\Lambda=240 \mathrm{~nm}, w_{a}=2.5 \mu \mathrm{m}$. (b) Normalized intensity of the electric field collected at plane I. (c) Normalized displacement profile of the mechanical mode we are interested in.

As an example, the normalized intensity profile of the subwavelength optical mode at $195 \mathrm{THz}$ (effective index is 2.4) and the displacement profile of the mechanical mode at $9.1 \mathrm{GHz}$ are shown in Figs 1 (b) and (c), respectively. The optical and mechanical mode profiles are collected at the I plane with $L_{a}=50 \mathrm{~nm}$. Low optical loss down to $2 \mathrm{~dB} / \mathrm{cm}$ using subwavelength gratings is achievable, reported in earlier works [22-25] and is not detailed here. The mechanical band diagram of the proposed waveguide is obtained using a Finite-Element-Method (FEM) model, considering the $1^{\text {st }}$ degenerate Brillouin zone, with the wavevector along the direction of light propagation $(z)$. The waveguide supports more than 30 mechanical modes with frequencies varying between a few $\mathrm{MHz}$ and $15 \mathrm{GHz}$. As an illustration, Fig. 2(a) shows the band structure of 8 modes of different kinds. The normalized displacement distributions of these mechanical modes (excited by the same mechanical power) are plotted in Fig. 2 (b). We can distinguish modes with flexural displacement concentrated in the nanoarms (Fig. 2 bI), or in entire structure (Fig. 2 b II, III) and modes with hybrid flexural-torsional displacement (Fig. 2 b V-VIII). The optoacoustic overlap is evaluated using $\eta=V \int_{V} \boldsymbol{u} \cdot \boldsymbol{E}_{p}^{*} \cdot \boldsymbol{E}_{s} d V /$ $\left[\int_{V}|u| d V \cdot \int_{V} \boldsymbol{E}_{p}^{*} \cdot \boldsymbol{E}_{s} d V\right]$, where $\boldsymbol{u}$ is the vectorial distribution of the displacement (all the vectorial quantity is labelled in bold) over the entire waveguide. The overlap values are indicated in the figures. A few of these modes present a considerable absolute displacement. However, only the mode with mainly transverse displacement along $\mathrm{x}$ in the core and nanoarms (mode IV, shown in Fig. 2(b)) allows strong optomechanical coupling with the TE optical mode. The angular frequency of this mechanical mode is $\Omega_{m} \sim 2 \pi \cdot 9 \mathrm{GHz}$. According to the energy conservation condition, the angular frequencies of the optical pump $\left(\omega_{p}\right)$ and Stokes $\left(\omega_{s}\right)$ are related by $\Omega_{m}=\omega_{p}-\omega_{s}$. Concurrently, the wavevector of the mechanical mode matching the momentum conservation condition can be calculated as $q=n_{g}\left(\omega_{p}-\omega_{s}\right) / c$. The fundamental optical Bloch mode of the optomechanical waveguide has a group index of $n_{g} \sim 4.6$ near $1550 \mathrm{~nm}$ wavelength. This yields $q<1000(2 \pi$. $\mathrm{m}^{-1}$ ) [16] and limits the interacting mode at the band edge.

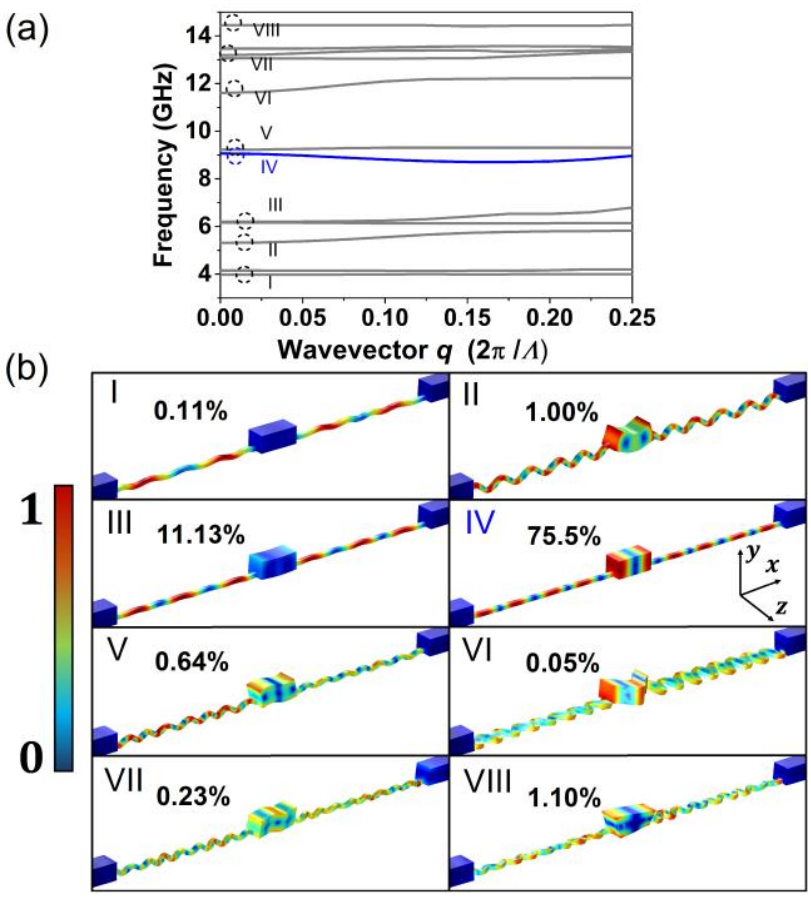

Fig. 2. (a) Band diagram of the mechanical modes of the proposed optomechanical waveguide with $w_{a}=50 \mathrm{~nm}$. The mode indicated with a blue curve is used later for the Brillouin gain evaluation. (b) Normalized displacement profile of some selected modes, including flexural modes (I-III), longitudinally strained mode (IV), and flexuraltorsional hybrid mode (V-VIII).

To assess the performance of the proposed optomechanical waveguide we use the Brillouin gain, $G_{m}$. $[10-12,15]$ From coupledmode theory it follows that the evolution of the power in the Stokes wave is governed by 


$$
\frac{d P_{s}}{d z}=-G_{m} P_{p} P_{s}+\alpha_{s} P_{s}
$$

being $P_{s}$ and $P_{p}$ the power in the pump and Stokes waves, respectively, and $\alpha_{s}$ the propagation loss of the Stokes waves. Assuming that the optical mode and mechanical mode (induced by the optical force) co-propagate along the waveguide, the Brillouin gain coefficient, with units of $\left(W^{-1} \cdot m^{-1}\right)$, can be calculated via $[10,15,26,27]$ :

$$
G_{m}=\frac{2 \omega_{p} Q_{m} S(\Omega)}{P_{a p} P_{a s} \Omega_{m}^{2} u_{m}^{2} \tilde{m}_{e f f}} \cdot \frac{1}{\Lambda}\left|\int f_{d, m b}(z) d A+\int f_{d, p e}(z) d V\right|^{2}
$$

with $S(\Omega)$ the Lorentzian spectrum of the Brillouin gain and $S\left(\Omega_{m}\right)=1$ is obtained with eigen acoustic frequency $\Omega_{m}$. In Eq. (2), $P_{a, i=p, s}=\left(\int \boldsymbol{E}_{i} \times \boldsymbol{H}_{i}^{*} \cdot \hat{z} d A\right) / 2$ is the time-average energy flux carried by the unnormalized optical pump $(i=p)$ and Stokes $(i=s)$ modes while $u_{m}$ gives the maximum value of the displacement. The effective linear mass density of the mechanical mode is $\widetilde{m}_{e f f}=$ $\frac{1}{\Lambda} \int \rho_{0}\left|u / u_{m}\right|^{2} d V$. The quality factor of the mechanical mode, $Q_{m}$, is related to the full width at half maximum (FWHM) of the gain spectrum, $\gamma_{m}$, through $Q_{m}=\Omega_{m} / \gamma_{m}$. It is limited by different loss mechanisms, described through their associated quality factors as:

$$
\frac{1}{Q_{m}}=\frac{1}{Q_{T E}}+\frac{1}{Q_{T V}}+\frac{1}{Q_{V E}}+\frac{1}{Q_{L}}
$$

Here, we consider the thermo-elastic loss $\left(Q_{T E}\right)$, the thermoviscous loss from surrounding air $\left(Q_{T V}\right)$, the viscoelastic loss $\left(Q_{V E}\right)$ and the loss from the acoustic leakage to the lateral silicon slab $\left(Q_{L}\right)$. The quality factor is defined via $Q_{i}=\Omega_{m} /\left(\gamma_{i}\right)$ where $\gamma_{i=T E, T V, V E, L} / 2$ is the decay rate of the mechanical mode. The mechanical quality factor changes nonlinearly with the length of the arms. For silicon nanostructures, the thermoelastic effect and the thermo-viscous effect typically yield quality factors of $Q_{T E} \sim 60000$ and $Q_{T V} \sim 3000$, respectively [28]. The viscoelastic loss of suspended silicon membrane is negligible due to the strain release through the liberation $[29,30]$. At atmospheric pressure, the maximum achievable mechanical quality factor is limited to $Q_{m} \sim 3000$ by the thermo-viscous loss induced by the surrounding air. However, to achieve this optimum $Q_{m}$, it is necessary to minimize mechanical leakage towards the lateral silicon slab. The $Q_{L}$ is mainly governed by the geometries of the waveguide and the nanoarms anchoring it to the lateral silicon slab. Note that we include 8- $\mu \mathrm{m}$-wide slab sections at the two sides of the structure to void any reflection artefact that may result in an overestimation on the quality factor.

The phonon confinement within the waveguide core is mainly governed by the filling factor in the arms, described as $\left(w_{a}\right.$. $\left.t_{a}\right) /\left(\Lambda \cdot t_{w g}\right)$. The lower the filling factor, the higher the confinement and the lower the leakage towards the lateral silicon slab. Maximum period length is fixed by subwavelength condition, while minimum value is determined by achievable feature size. Thus, we set $t_{a}=50 \mathrm{~nm}, t_{w g}=220 \mathrm{~nm}, w_{w g}=500 \mathrm{~nm}$ and $\Lambda=240$ $\mathrm{nm}, w_{a}=2.5 \mu \mathrm{m}$, as a compromise between high phonon confinement and achievable minimum feature size. In Fig. (3) we show the mechanical quality factor and the Brillouin gain, calculated as a function of the arm length $\left(L_{a}\right)$. A remarkable $Q_{m}$ of up to 1200 can be obtained for an arm length of $25 \mathrm{~nm}$.

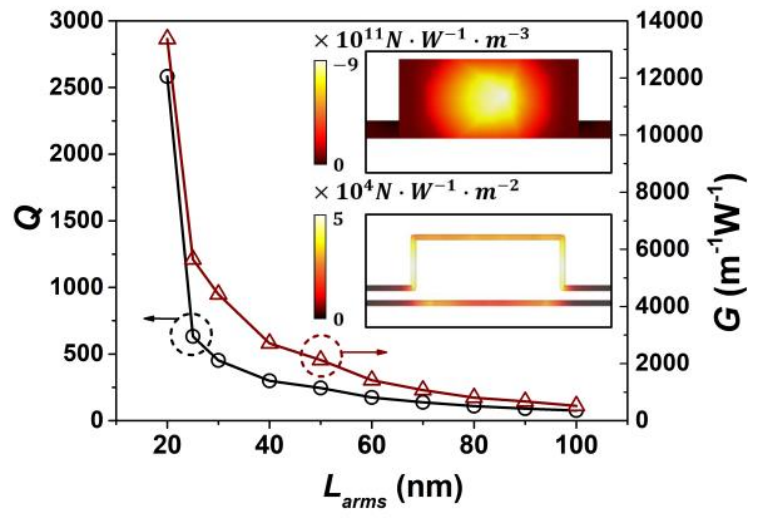

Fig. 3. The quality factor (black) of the mechanical mode and the overall Brillouin gain (red) as a function of the length of silicon arms $L_{\text {arms }}$. Upper panel (inset) is the modulus of normalized force density regarding to the photoelastic effect $f_{n d, p e}$, while the lower one is the boundary motion-induced force density along the interface. Both density distribution is taken at the cross-section I that shown in Fig. 1.

The Brillouin gain is calculated via Equ. (2), with force density associated with the moving boundary effect $[10,18] \boldsymbol{f}_{d, m b}=$ $\boldsymbol{u}^{*}\left(\delta \varepsilon \boldsymbol{E}_{p, \|}^{*} \cdot \boldsymbol{E}_{s, \|}-\delta \varepsilon^{-1} \boldsymbol{D}_{p, \perp}^{*} \cdot \boldsymbol{D}_{s, \perp}\right) / 4$ and force density produced by the photoelastic effect $\boldsymbol{f}_{d, p e}=-\varepsilon_{0} \varepsilon_{1}^{2} \boldsymbol{T}_{p e} \cdot \boldsymbol{T}_{s} \cdot \boldsymbol{E}_{p}^{*} \cdot \boldsymbol{E}_{s} / 4$ [10]. Here $\boldsymbol{D}$ is the electric displacement field and $\delta \varepsilon=\varepsilon_{1}-\varepsilon_{2}$ and $\delta \varepsilon^{-1}=\varepsilon_{1}{ }^{-1}-\varepsilon_{1}{ }^{-2}$ describe the permittivity difference between core $\varepsilon_{1}$ and surrounding $\varepsilon_{2}$. The $\boldsymbol{T}_{p e}$ and $\boldsymbol{T}_{s}$ represent photoelastic tensor and strain tensor induced by the mechanical waves. In Equ. (2), $d A$ and $d V$ indicates a surface integral and volume integral, respectively. As shown in Fig. 3, the Brillouin gain follows a similar trend as quality factor, with a sharp increase for arm lengths below $30 \mathrm{~nm}$. For an arm length of $20 \mathrm{~nm}$, the Brillouin gain reaches $10000(\mathrm{~W} \cdot \mathrm{m})^{-1}$. For an arm length of $50 \mathrm{~nm}$, compatible with electron-beam lithography, the Brillouin gain is $2000(W \cdot m)^{-1}$. This gain value compares favorably with previously proposed silicon membranes with periodic silicon arms [19], while obviating the need for transversal Bragg phononic mirrors. Increasing the air gap in the nanoarms reduces Brillouin linewidth reaching $34 \mathrm{MHz}$ $\left(Q_{m}=260\right)$ for a duty cycle of $20 \%$.

The distribution of modulus of these two force densities $f_{n d, i}=$ $\frac{f_{d, i}}{\sqrt{P_{a p} P_{a s}} \cdot u_{m}}$ where $i=p e, m b$, are shown in the inset of Fig. 3 for an arm length of $L_{a}=50 \mathrm{~nm}$. Both density distributions are taken at the cross-section I in Fig. 1. The $f_{n d, p e}$ presents a distribution similar to that of the optical mode shown in Fig. 1, since it is directly related to the vectorial product of the strain profile and the electric field $E_{x}$. Conversely, the $f_{n d, m b}$ exists at waveguide boundaries, associating with the tangential electric field components ( $E_{z}$ in this case). Taking the linear integral of $f_{n d, p e}$ along the $x$ direction, we find that it has values with same order of magnitude but opposite signs to $f_{n d, m b}$. These two forces are driven along different direction, partially cancelling each other, reducing the overall Brillouin gain. This result is in agreement with previous studies on the interactions between electrostriction and radiation pressure in silicon waveguides [11,15]. The Brillouin gain could be further increased by achieving constructive interaction between the photoelastic and the moving-boundary effects, e.g. by geometry optimization or interaction between different optical modes [15, 17]. 
We have as well studied the impact of the silicon thickness on the Brillouin gain and mechanical quality factor. In this case, we fixed the thickness of the nanoarms to $t_{a}=50 \mathrm{~nm}$ and the length to $L_{a}=$ $50 \mathrm{~nm}$ (compatible with electron-beam lithography). As observed in Fig. 4 (a), both the gain and quality factor increase with a thicker Si layer, up to a thickness of $300 \mathrm{~nm}$. For a silicon thickness of 290 $\mathrm{nm}$, the Brillouin gain reaches $3000(\mathrm{~W} \cdot \mathrm{m})^{-1}$. When the silicon thickness is larger than $300 \mathrm{~nm}$, the maximum displacement shifts from the waveguide core to the nanoarms, substantially increasing the mechanical quality factor but is simultaneously responsible for a reduced photon-phonon overlap. It is a direct indication that, the figure of merit $G / Q$ drops sharply for waveguide thicknesses above $300 \mathrm{~nm}$ (inset). To illustrate this behavior, we plot the displacement profile of the mechanical mode for a silicon thickness of $t_{w g}=280 \mathrm{~nm}$, approximately maximizing the gain, and of $t_{w g}=$ $310 \mathrm{~nm}$, in Figs. 4 (b) and 4(c), respectively.

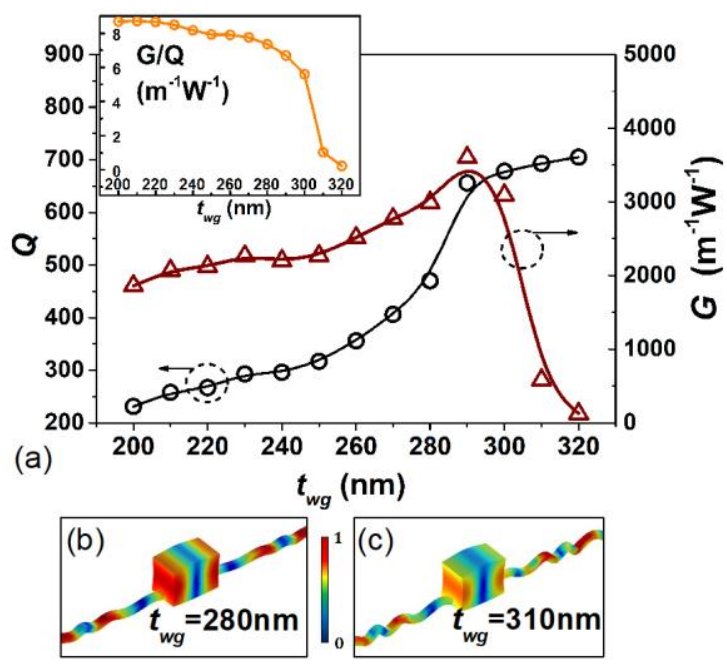

Fig. 4. (a) The quality factor (black) of the mechanical mode and the overall Brillouin gain (red) as a function of the thickness of silicon rib $t_{w g}$. The dimension of the nanoarms $t_{a}$ and $L_{a}$ are both fixed at $50 \mathrm{~nm}$. Inset is gain over the mechanical $Q$ with varying $t_{w g}$. (b) and (c) are the normalized displacement profile of the mechanical mode with $t_{w g}=$ $280 \mathrm{~nm}$ and $t_{w g}=310 \mathrm{~nm}$, respectively.

In summary, we have proposed and analyzed a new strategy to optimize Brillouin scattering in silicon membrane waveguides. The proposed waveguide geometry confines mechanical and optical modes by subwavelength softening and subwavelength index engineering, respectively, and allows to simultaneously enlarge the photon-phonon interaction and improves the quality factor of the mechanical mode. Our calculations show that the proposed optomechanical waveguide could provide a Brillouin gain exceeding $3000(\mathrm{~W} \cdot \mathrm{m})^{-1}$ for a minimum feature size of $50 \mathrm{~nm}$, compatible with electron-beam lithography, and up to 10000 (W · $\mathrm{m})^{-1}$ for minimum feature size of $20 \mathrm{~nm}$. These results showcase the great potential of subwavelength engineering to shape mechanical and optical modes with silicon. We believe these results will expedite the use of subwavelength-lattice waveguides to engineer photon-phonon interaction in silicon.
Funding. Agence Nationale de la Recherche (ANR) (MIRSPEC ANR17-CE09-0041, BRIGHT ANR-18-CE24-0023-01); European Research Council (ERC) (No. 715939, Nanophennec)

Disclosures. The authors declare no conflicts of interest.

\section{References}

1. G. P. Agrawal, Nonlinear Fiber Optics., 5th ed., Rochester, New York, USA, (2013).

2. B. J. Eggleton, C. G. Poulton, P. T. Rakich, M. J. Steel, and G. Bahl, Nat. Photon. 13, 664 (2019).

3. R. V. Laer, R. Baets, and D. Van Thourhout, Phys. Rev. A 93, 053828 (2016).

4. R Pant, C. G. Poulton, D. Choi, H. Mcfarlane, S. Hile, E. Li, L. Thevenaz, B. Luther-Davies, S. J. Madden, and B. J. Eggleto, Opt. Exp. 19, 8285 (2011).

5. A. Naeini, D. Van Thoughout, R. Baets, and R. Laer, Optica 6, 213 (2019).

6. G. Arregui, N.D. Lanzillotti-Kimura, C.M. Sotomayor-Torres, and P.D. García, Phys. Rev. Lett. 122, 043903 (2019).

7. F. Kargar, B. Debnath, J.-P. Kakko, A. Säynätjoki, H. Lipsanen, D. L. Nika, R. K. Lake, and A. A. Balandin, Nat. Comm. 7, 13400 (2016).

8. F.R. Lamberti, Q. Yao, L. Lanco, D. T. Nguyen, M. Esmann, A. Fainstein, P. Sesin, S. Anguiano, V. Villafañe, A. Bruchhausen, P. Senellart, I. Favero, and N. D. Lanzillotti-Kimura, Opt. Exp. 25, 24437 (2017).

9. M. Esmann, F. R. Lamberti, A. Harouri, L. Lanco, I. Sagnes, I. Favero, G. Aubin, C. Gomez-Carbonell, A. Lemaître, O. Krebs, P. Senellart, and N. D. LanzillottiKimura, Optica 6, 854 (2019).

10. P. T. Rakich, C. Reinke, R. Camacho, P. Davids, and Z. Wang, Phys. Rev. X 2, 011008 (2012).

11. P. T. Rakich, P. Davids, and Z. Wang, Opt. Exp. 18, 14439 (2010).

12. W. Qiu, P. T. Rakich, H. Shin, H. Dong, M. Soljǎci'c, and Z. Wang, Opt. Exp. 21, 31402 (2013).

13. R. Van Laer, B. Kuyken, D. Thourhout, and R. Baets, Nat. Photon. 9, 199 (2015).

14. C. J. Sarabalis, J. T. Hill, and A. Safavi-Naeini, APL Photon. 1, 071301 (2016).

15. C. Wolff, M. J. Steel, B. J. Eggleton and C. G. Poulton, Phys. Rev. A 92, 013836 (2015).

16. E. A. Kittlaus, H. Shin and P. T. Rakich, Nat. Photon. 10, 463 (2015).

17. N. T. Otterstrom, R. O. Behunin, E. A. Kittlaus, Z. Wang, and P. T. Rakich, Science 360, 1113 (2016).

18. Steven G. Johnson, M. Ibanescu, M. A. Skorobogatiy, O. Weisberg, J. D. Joannopoulos, and Y. Fink, Phys. Rev. A 65, 066611 (2002).

19. M. K. Schmid, C. G. Poulton, G. Z. Mashanovich, G. T. Reed, B. J. Eggleton, and M. J. Steel, Opt. Exp. 27, 4976 (2019).

20. R. Zhang, J. Sun, J. of Light. Tech. 35, 14 (2017).

21. T. Baba, Nat. Photon. 2, 465 (2008).

22. P. Cheben, D-X. Xu, S. Janz, and A. Densmore, Opt. Exp. 14, 4695 (2019).

23. P. Cheben, P. J. Bock, J. H. Schmid, J. Lapointe, S. Janz, D. Xu, A. Densmore, A. Delâge, B. Lamontagne, and Trevor J. Hall, Opt. Lett. 35, 2526 (2010).

24. R. Halir, P. J. Bock, P. Cheben, A. Ortega-Moñux, C. Alonso-Ramos, J. H. Schmid, J. Lapointe, D.-X. Xu, J. G. Wangüemert-Pérez, I. Molina-Fernández, and S. Janz, Las. Photon. Rev. 9, 25 (2015).

25. P. Cheben, R. Halir, J. Schmid, H. Atwater, and D. Smith, Nature 560, 565 (2018).

26. G. S. Wiederhecker, P. Dainese, T. Alegre, APL Photon. 4, 071101 (2019).

27. M. Eichenfield, J. Chan, R. M. Camacho, K. J. Vahala, O. Painter, Nature 462, 78 (2009).

28. COMSOL, COMSOL Multiphysics v5.3.

29. H. Kahn, N. Jing, M. Huh, and A.H. Heuer, J. Mater. Res 21, 209 (2006).

30. A. Zimmer, R. Nawrodt, D. Heinert, C. Schwarz, M. Hudl, T. Koettig, W. Vodel, A. Tunnermann and P. Seidel, Journal of Physics: Conference Series 92, 012095 (2007). 


\section{References (for reviewing)}

1. G. P. Agrawal, Nonlinear Fiber Optics., 5th ed., Rochester, New York, USA, (2013).

2. B. J. Eggleton, C. G. Poulton, P. T. Rakich, M. J. Steel, and G. Bahl, "Brillouin integrated photonics," Nat. Photon. 13, 664 (2019).

3. R. V. Laer, R. Baets, and D. Van Thourhout, "Unifying Brillouin scattering and cavity optomechanics," Phys. Rev. A 93, 053828 (2016).

4. R Pant, C. G. Poulton, D. Choi, H. Mcfarlane, S. Hile, E. Li, L. Thevenaz, B. Luther-Davies, S. J. Madden, and B. J. Eggleto, "On-chip stimulated Brillouin scattering," Opt. Exp. 19, 8285 (2011).

5. A. H. Safavi-Naeini, D. Van Thoughout, R. Baets, and R. V. Laer, "Controlling phonons and photons at the wavelength scale integrated photonics meets integrated phononics," Optica 6, 213 (2019).

6. G. Arregui, N.D. Lanzillotti-Kimura, C.M. Sotomayor-Torres, and P.D. García, "Anderson Photon-Phonon Colocalization in Certain Random Superlattices," Phys. Rev. Lett. 122, 043903 (2019).

7. F. Kargar, B. Debnath, J.-P. Kakko, A. Säynätjoki, H. Lipsanen, D. L. Nika, R. K. Lake, and A. A. Balandin, "Direct observation of confined acoustic phonon polarization branches in free-standing semiconductor nanowires," Nat. Comm. 7, 13400 (2016).

8. F.R. Lamberti, Q. Yao, L. Lanco, D. T. Nguyen, M. Esmann, A. Fainstein, P. Sesin, S. Anguiano, V. Villafañe, A. Bruchhausen, P. Senellart, I. Favero, and N. D. Lanzillotti-Kimura, "Optomechanical properties of GaAs/AlAs micropillar resonators operating in the $18 \mathrm{GHz}$ range," Opt. Exp. 25, 24437 (2017).

9. M. Esmann, F. R. Lamberti, A. Harouri, L. Lanco, I. Sagnes, I. Favero, G. Aubin, C. Gomez-Carbonell, A. Lemaître, O. Krebs, P. Senellart, and N. D. LanzillottiKimura, "Brillouin scattering in hybrid optophononic Bragg micropillar resonators at $300 \mathrm{GHz}$," Optica 6, 854 (2019).

10. P. T. Rakich, C. Reinke, R. Camacho, P. Davids, and Z. Wang, "Giant Enhancement of Stimulated Brillouin Scattering in the Subwavelength Limit," Phys. Rev. X 2, 011008 (2012).

11. P. T. Rakich, P. Davids, and Z. Wang, "Tailoring optical forces in waveguides through radiation pressure and electrostrictive forces," Opt. Exp. 18, 14439 (2010).

12. W. Qiu, P. T. Rakich, H. Shin, H. Dong, M. Soljačić, and Z. Wang, "Stimulated Brillouin scattering in nanoscale silicon step-index waveguides," Opt. Exp. 21, 31402 (2013).

13. R. Van Laer, B. Kuyken, D. V. Thourhout, and R. Baets, "Interaction between light and highly confined hypersound in a silicon photonic nanowire," Nat. Photon. 9, 199 (2015).

14. C. J. Sarabalis, J. T. Hill, and A. H. Safavi-Naeini, "Guided acoustic and optical waves in silicon-on-insulator for Brillouin scattering and optomechanics," APL Photon. 1, 071301 (2016).

15. C. Wolff, M. J. Steel, B. J. Eggleton and C. G. Poulton, "Stimulated Brillouin scattering in integrated photonic waveguides Forces, scattering mechanisms and coupled-mode analysis," Phys. Rev. A 92, 013836 (2015).

16. E. A. Kittlaus, H. Shin and P. T. Rakich, "Large Brillouin amplification in silicon," Nat. Photon. 10, 463 (2015).

17. N. T. Otterstrom, R. O. Behunin, E. A. Kittlaus, Z. Wang, and P. T. Rakich, “A Silicon Brillouin laser," Science 360, 1113 (2016).

18. Steven G. Johnson, M. Ibanescu, M. A. Skorobogatiy, O. Weisberg, J. D. Joannopoulos, and Y. Fink, "Perturbation theory for Maxwell's equations with shifting material boundaries," Phys. Rev. A 65, 066611 (2002).

19. M. K. Schmid, C. G. Poulton, G. Z. Mashanovich, G. T. Reed, B. J. Eggleton, and M. J. Steel, "Suspended mid-infrared waveguides for Stimulated Brillouin Scattering," Opt. Exp. 27, 4976 (2019).

20. R. Zhang, J. Sun, "Design of Silicon Phoxonic Crystal Waveguides for Slow Light Enhanced Forward Stimulated Brillouin Scattering," J. of Light. Tech. 35, 14 (2017).

21. T. Baba, "Slow light in photonic crystals," Nat. Photon. 2, 465 (2008).

22. P. Cheben, D-X. XU, S. Janz, and A. Densmore, "Design of Silicon Phoxonic Crystal Waveguides for Slow Light Enhanced Forward Stimulated Brillouin Scattering," Opt. Exp. 14, 2006 (2019).
23. P. Cheben, P. J. Bock, J. H. Schmid, J. Lapointe, S. Janz, D. Xu, A. Densmore, A. Delâge, B. Lamontagne, and Trevor J. Hall, "Refractive index engineering with subwavelength gratings for efficient microphotonic couplers and planar waveguide multiplexers," Opt. Lett. 35, 2526 (2010).

24. R. Halir, P. J. Bock, P. Cheben, A. Ortega-Moñux, C. Alonso-Ramos, J. H. Schmid, J. Lapointe, D.-X. Xu, J. G. Wangüemert-Pérez, I. Molina-Fernández, S. Janz, "Waveguide sub-wavelength structures a review of principles and applications," Las. Photon. Rev. 9, 25 (2015).

25. P. Cheben, R. Halir, J. H. Schmid, H. A. Atwater, D. R. Smith, "Subwavelength integrated photonics," Nature 560, 565 (2018).

26. G. S. Wiederhecker, P. Dainese, and T. P. M. Alegre, "Brillouin optomechanics in nanophotonic structures," APL Photon. 4, 071101 (2019).

27. M. Eichenfield, J. Chan, R. M. Camacho, K. J. Vahala, and O. Painter, "Optomechanical crystals," Nature 462, 78 (2009).

28. COMSOL, COMSOL Multiphysics v5.3.

29. H. Kahn, N. Jing, M. Huh, and A.H. Heuer, "Growth stresses and viscosity of thermal oxides on silicon and polysilicon," J. Mater. Res 21, 209 (2006).

30. A. Zimmer, R. Nawrodt, D. Heinert, C. Schwarz, M. Hudl, T. Koettig, W. Vodel, A. Tunnermann and P. Seidel, "Mechanical losses in low loss materials studied by cryogenic resonant acoustic spectroscopy of bulk materials (CRA spectroscopy)," Journal of Physics: Conference Series 92, 012095 (2007). 